\title{
The Effects Of Sevoflurane On The Progression And Cisplatinum Sensitivity Of Cervical Cancer Cells
}

\author{
Fang Xue \\ Yichi Xu \\ Yizuo Song \\ Wenwen Zhang $\mathbb{1}$ \\ Ruyi Li \\ Xueqiong Zhu (D)
}

Department of Obstetrics and Gynecology, The Second Affiliated Hospital of Wenzhou Medical University, Wenzhou 325027, People's Republic of China
Correspondence: Ruyi Li; Xueqiong Zhu Department of Obstetrics and Gynecology, The Second Affiliated Hospital of Wenzhou Medical University, No. 109 Xueyuan Xi Road, Wenzhou, Zhejiang 325027, People's Republic of China

Tel +86 57788002796 (office);

Fax +8657788002796

Email ruyili877I@I26.com;

zjwzzxq@163.com
This article was published in the following Dove Press journal:

Drug Design, Development and Therapy

Objective: To investigate the effect of sevoflurane on the progression of cervical cancer cells, and to explore its effect on the cisplatinum (DDP) sensitivity in cervical cancer cells and underlying mechanism.

Methods: Siha and Hela cervical cancer cells were cultured and treated with 3\% sevoflurane, $10 \mu \mathrm{mol} / \mathrm{L}$ DDP, or the co-treatment of sevoflurane and DDP, respectively. Cell proliferation was evaluated by the CCK 8 assay. Cell apoptosis was assessed by flow cytometry. Cell migration was detected by wound healing assay. The expression of B-cell lymphoma-2 (BCL-2), B-cell lymphoma-2 associated X (BAX), Ezrin, matrix metalloproteinase 2 (MMP2), lung resistancerelated protein (LRP), multiple drug resistance protein 1 (MRP1), glutathione-S-transferase- $\pi$ (GST- $\pi$ ), and P glycoprotein (P-gp) protein was determined by Western blotting.

Results: After treated with sevoflurane, cell proliferation and migration rate in Siha and Hela cells were significantly elevated, while cell apoptosis was decreased. In addition, the expression of migration-related protein Ezrin and MMP2 was increased accordingly, apoptotic-related protein BCL-2 expression was also increased while BAX protein expression was decreased after sevoflurane treatment. The proliferation, migration rate, and apoptosis of Siha and Hela cells in sevoflurane plus DDP group were not significantly different with those in DDP group. There was no significant difference in apoptotic-related protein, migrationrelated protein, and drug resistance-associated proteins expression between DDP treatment group and combined treatment group.

Conclusion: Sevoflurane promotes the progression but has no effect on the cisplatinum sensitivity in cervical cancer cells.

Keywords: sevoflurane, cisplatinum, proliferation, migration, apoptosis, Siha, Hela

\section{Introduction}

Cervical cancer is the most common cancer among women in developing countries and the third leading cause of cancer mortality, with global cases and deaths estimated to be 13,170 and 4250 in 2019 , respectively. ${ }^{1}$ While surgery is considered the preferred treatment for patients with early cervical cancer, chemotherapy is the optimal treatment for patients with advanced, recurrent, and metastatic cervical cancer. ${ }^{2,3}$ Platinum-based drugs, which effectively eradicate tumor cells, are still considered as the first-line anticancer drugs in numerous human cancers including cervical cancer, but the resistance of cancer cells to DDP seriously hinders its clinical application. ${ }^{4}$

Sevoflurane, a colorless, transparent and non-irritating volatile anesthetic, has been widely used in the surgical process of malignancy tumors including cervical 
cancer. In recent years, increasing evidence suggests that this inhaling anesthetics have impact on the biological behavior of cancer cells. ${ }^{5-7}$ Additionally, previous studies reported that sevoflurane has a different effect on the chemosensitivity of cisplatinum in distinct cancer cells. ${ }^{8-10}$ Sevoflurane had no influence on DDP sensitivity in mouse brain cells. ${ }^{8}$ However, it enhanced DDP sensitivity in nonsmall cell lung cancer (A549) cells, ${ }^{11}$ while in renal cell carcinoma (RCC4) cells ${ }^{9}$ and Ehrlich ascites tumor cells, ${ }^{10}$ it attenuated the sensitivity of cells to DDP. To date, there was no published report on whether sevoflurane has effect on DDP sensitivity in cervical cancer cells, and whether patients who show resistance to DDP chemotherapy are more sensitive to sevoflurane, as well.

In the present study, the effects of sevoflurane on the proliferation, migration and apoptosis of cervical squamous cancer Siha cells and adenocarcinoma HeLa cells and underlying mechanism were investigated. In addition, the effects of sevoflurane on the DDP sensitivity in cervical cancer cells were explored after comparing the cell viability, biological behavior, apoptotic-related protein, migration-related protein, and drug resistance-associated proteins expression between DDP treatment group and sevoflurane combined with DDP treatment group.

\section{Materials And Methods Cell Lines And Cell Culture}

Human cervical cancer cells Siha and HeLa were purchased from European Collection of Authenticated Cell Cultures. Siha cell was established from fragments of primary tissue sample obtained after surgery from a Japanese patient. HeLa cell was a type of cervical adenocarcinoma cell line. Cells were cultured in Dulbecco's modified Eagle's Medium (DMEM, Gibco, USA) containing 10\% fetal bovine serum. All the cells were incubated in a humidified atmosphere containing $5 \% \mathrm{CO}_{2}$ at $37^{\circ} \mathrm{C}$ for growth.

\section{Sevoflurane And Combined Treatment}

Cells were split and seeded at equal cell density in $30 \mathrm{~mm}^{2}$ Petri dishes (VWR, Leicestershire, UK) and 6, 24 and 96-well plates, then incubated at $37^{\circ} \mathrm{C}$ in a humidified atmosphere containing $5 \% \mathrm{CO}_{2}$ for growth. When the cultures reached $70 \%-80 \%$ confluence, the plates were placed in a $2 \mathrm{~L}$ purposely built gas chamber with inlet and outlet connectors. Sevoflurane mixed with $5 \% \mathrm{CO}_{2}$ balanced with air was delivered into the chamber by a vaporizer (Sevoflurane; Abbott, Abbot Park, IL, USA) at a rate of $2 \mathrm{~L} / \mathrm{min}$ for $5 \mathrm{~min}$ until the desired gas dose was reached. Sevoflurane concentrations in the chamber were monitored using an anesthetic monitor (Datex-Ohmeda, Stirling, UK) at the outlet part of chamber. When the sevoflurane concentration of the chamber reached $3 \%$, the gas delivery was stopped and the inlet and outlet were clamped, respectively. The chamber was then transferred to a $37^{\circ} \mathrm{C}$ incubator (Galaxy $\mathrm{R} \mathrm{CO}_{2}$ chamber; New Brunswick Scientifc, Enfeld, USA) to expose the cells to 3\% sevoflurane for $2 \mathrm{hrs}$. In clinical operations, the minimum effective concentration of less than $4 \%$ is usually used to maintain the anesthetic state. In our preliminary studies, the cell viability of Hela cells was increased after $1 \%, 2 \%$ or $3 \%$ sevoflurane treatment and the effect of sevoflurane on cell viability was concentration-dependence. Therefore, sevoflurane at a concentration of $3 \%$ was chosen in the present study to treat cells. The half maximum inhibitory concentration (IC50) of DDP on SiHa cells was $18 \mu \mathrm{mol} / \mathrm{L}$ and Hela cells was $18.3 \mu \mathrm{mol} / \mathrm{L}$ (Supplementary Figure 1). Referring to IC50 value, $1 \mathrm{mM}$ reserve solution (Calbiochem, USA, CAT 232120) was diluted to $10 \mu \mathrm{mol} / \mathrm{L}$ with DMEM to make a DDP- supplemented media. Cells were divided into four treatment groups: control group, sevoflurane group, DDP group, and a combination of sevoflurane and DDP group (Table 1). The control group was treated with $95 \%$ air $/ 5 \% \mathrm{CO}_{2}$ at $2 \mathrm{~L} / \mathrm{min}$ for $2 \mathrm{hrs}$. The sevoflurane group was treated with $3 \%$ sevoflurane mixed with $95 \%$ air $/ 5 \% \mathrm{CO}_{2}$ at $2 \mathrm{~L} / \mathrm{min}$ for $2 \mathrm{hrs}$. The DDP group was treated with $10 \mu \mathrm{mol} / \mathrm{L}$ DDP and immediately exposed to $95 \%$ air $/ 5 \% \mathrm{CO}_{2}$ at $2 \mathrm{~L} / \mathrm{min}$ for $2 \mathrm{hrs}$. The sevoflurane and DDP group were treated with $10 \mu \mathrm{mol} / \mathrm{L}$ DDP and immediately exposed to $3 \%$ sevoflurane mixed with $95 \%$ air $/ 5 \% \mathrm{CO}_{2}$ at $2 \mathrm{~L} / \mathrm{min}$ for $2 \mathrm{hrs}$. In order to be closer to the use of clinical medicines, we did not change the medium after gas exposure. Then, each group of cells were moved into a $37^{\circ} \mathrm{C}$ incubator for $24 \mathrm{hrs}$ to subsequent experiments.

\section{Cell Viability Assay}

To evaluate cell viability rate, $1 \times 10^{4}$ cells were plated in 96-well plates and cultured for $24 \mathrm{hrs}$ for the cells to

Table I Four Treatment Groups

\begin{tabular}{|l|l|l|}
\hline Treatment Groups & Concentration & Exposure Time, Hour \\
\hline Control & & \\
Sevoflurane & $3 \mathrm{vol} \%$ & 2 \\
DDP & $10 \mu \mathrm{mol} / \mathrm{L}$ & \\
Sevoflurane+DDP & $3 \mathrm{vol} \%+10 \mu \mathrm{mol} / \mathrm{L}$ & 2 \\
\hline
\end{tabular}

Notes: The Sevoflurane + DDP group were treated with $10 \mu \mathrm{mol} / \mathrm{L}$ DDP and immediately exposed to $3 \%$ sevoflurane mixed with $95 \%$ air $/ 5 \% \mathrm{CO}_{2}$ at $2 \mathrm{~L} / \mathrm{min}$ for $2 \mathrm{hrs}$, then moved into a $37^{\circ} \mathrm{C}$ incubator for $24 \mathrm{hrs}$. 
adhere. After DDP or/and sevoflurane treatment, the cells were cultured in a $\mathrm{CO}_{2}$ incubator for another $24 \mathrm{hrs}$. Thereafter, Cell Counting Kit-8 (CCK-8, Sigma, USA) was used to analyze cell viability and the number of viable cells was assessed by measurement of absorbance at 450 nm using a Microplate Reader (Bio Tek Instruments, Winooski, VT, USA) after 2 hrs incubation. The experiments were performed at least three times for the final analyses.

\section{Apoptosis Analysis By Flow Cytometry}

To evaluate cell apoptosis rate, $1 \times 10^{5}$ cells were plated in 6-well plates and culture for $24 \mathrm{hrs}$. Apoptosis was assessed using the Annexin V-FITC/PI Apoptosis Detection Kit (BD Pharmingen, CA, USA) apoptosis detection kit (BD, Franklin Lakes, NJ, USA) according to the manufacturer's instructions. After sevoflurane or/ and DDP treatment and continued culture for $24 \mathrm{hrs}$, cells were labeled with $5 \mu \mathrm{L}$ Annexin V-FITC and PI in dark at room temperature for $15 \mathrm{~min}$. The early apoptosis was determined based on the percentage of cells with Annexin V+/PI-, whereas the late apoptosis was that of cells with Annexin V+/PI+. The total number of early apoptosis plus late apoptosis is considered to be the ultimate apoptosis rate of the cells. The percentage of apoptotic cells analyzed by flow cytometry (Beckman Coulter, Fullerton, CA, USA). The experiments were performed at least three times for the final analyses.

\section{Wound Healing Assay}

To evaluate the migration ability of cancer cells, $1 \times 10^{5}$ cells were plated in 6-well plates and cultured to a fully fused state. After DDP or/and sevoflurane treatment, an artificial gap was created by scraping with a sterile plastic pipette tip $(200 \mu \mathrm{L})$. By a phase-contrast microscope and digital camera (Olympus CK30, Tokyo, Japan), migrating area from the initial wound was measured from images taken. Five randomly selected scratch areas from each well were analyzed in $0 \mathrm{hr}$ and $24 \mathrm{hrs}$ treatment using ImageJ 1.35 (NIH, Bethesda, MD, USA) software. The experiments were performed at least three times for the final analyses.

\section{Western Blot Analysis}

Cells were cultured in 6-well plate ( $1 \times 10^{6}$ cells per well). After sevoflurane or/and DDP treatment and an additional $24 \mathrm{hrs}$ cultured, the proteins expression of $\alpha$-tubulin, $\beta$-actin, BCL-2,
BAX, Ezrin, MMP2, LRP, MRP1, GST- $\pi$, and P-gp in cells was fractionated with sodium dodecyl sulfate-polyacrylamide gel electrophoresis, followed by transferring onto Polyvinylidene fluoride (PVDF) membrane. The membranes were incubated overnight at $4^{\circ} \mathrm{C}$ with the primary antibodies as following: anti-Ezrin (1:1000, Cell Signaling Technology, Denvers, MA, USA), anti-MMP2 (1:1000, Cell Signaling Technology, Denvers, MA, USA), anti- $\alpha$-Tubulin (1:1000, Cell Signaling Technology, Denvers, MA, USA), anti-BCL-2 (1:1000, Cell Signaling Technology, Denvers, MA, USA), anti-BAX (1:1000, Cell Signaling Technology, Denvers, MA, USA), anti- $\beta$-actin (1:3000, Cell Signaling Technology, Denvers, MA, USA) and anti-LRP (1:1000, Abcam, San Francisco, CA, USA), anti-MRP1 (1:1000, Abcam, San Francisco, CA, USA), anti-GST- $\pi$ (1:1000, Abcam, San Francisco, CA, USA), anti-P-gp (1:1000, Abcam, San Francisco, CA, USA). After washed with Tris-buffered saline with Tween (TBST), the membranes were incubated with horseradish peroxidase-conjugated goat anti-rabbit IgG (1:2000, Biosharp) or rabbit anti-mouse IgG secondary antibody $\left(1: 4000\right.$, Biosharp) at $37^{\circ} \mathrm{C}$ for $1.5 \mathrm{hrs}$. Protein signals were detected using enhanced chemiluminescence reagent (Millipore, USA). The intensity of proteins was quantified by densitometry using Bio-Rad Quantity One software (Bio-Rad, Hercules, USA). The experiments were performed at least three times.

\section{Statistical Analysis}

Data analysis was performed with Statistical Product and Service Solutions (SPSS) 19.0 statistical software (IBM Corporation, Armonk, NY, USA). Data were expressed as mean \pm standard deviation. Differences among groups were assessed using one-way ANOVA, followed by least significance difference method. Differences were considered statistically significant at a 2-sided $P$-value $<0.05$.

\section{Results}

\section{The Effect Of Sevoflurane And}

Sevoflurane Combined With DDP On The Cell Proliferation Rate Of Cervical

\section{Cancer Cells}

As shown in Figure 1, compared with the control group, the cell viability of Siha and Hela cells was increased after $3 \%$ sevoflurane treatment, while the cell viability was significantly decreased after DDP treatment. Whereas, the cell viability of Siha and Hela cells was no significant 


\section{Siha}

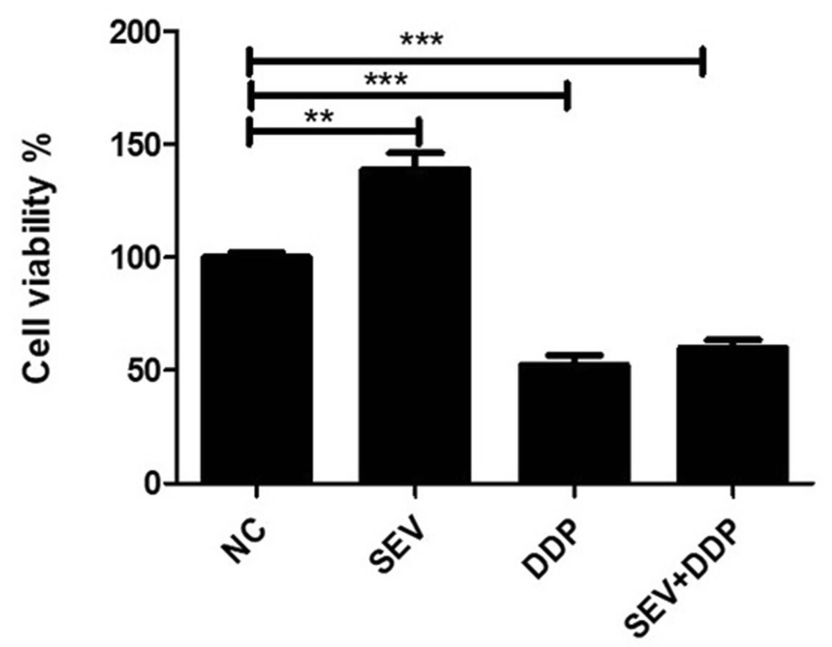

Hela

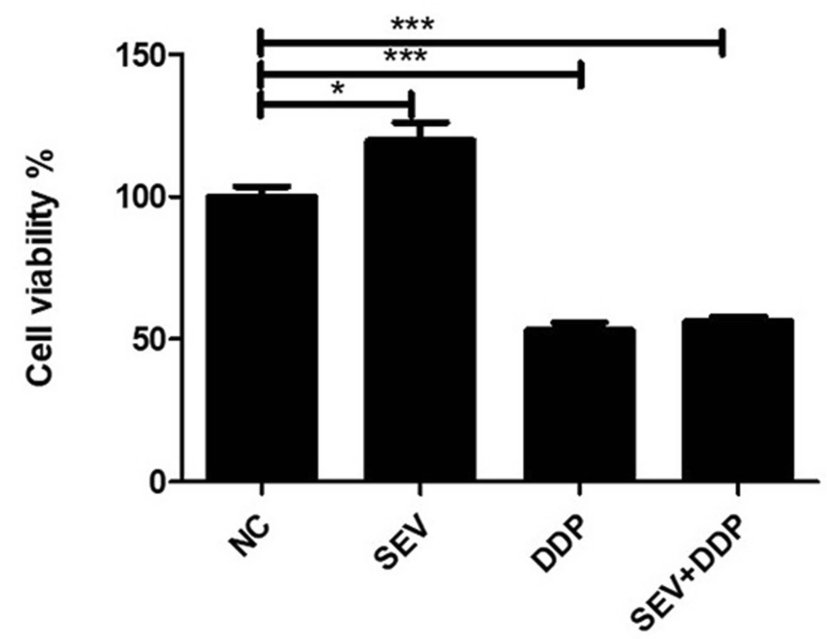

Figure I The effect of sevoflurane and sevoflurane combined with cisplatin (DDP) on the cell proliferation rate of cervical cancer cells. Siha and Hela cells were treated with sevoflurane, DDP, or the co-treatment of sevoflurane and DDP. The proliferation rate was evaluated by CCK 8 assay. The bars represent the cell viability rates of proliferation in each group. Data are shown as means and standard deviation of three independent experiments. $* P<0.05, * * P<0.01$, $* * * P<0.001$.

difference between the DDP group and sevoflurane combined with DDP group. These results indicated that sevoflurane promoted the proliferation of cells, but it did not affect the inhibition of DDP on the proliferation of cervical cancer cells.

\section{The Effect Of Sevoflurane And}

\section{Sevoflurane Combined With DDP On}

\section{The Cell Apoptosis And Apoptotic-} Related Protein BCL-2 And BAX In

\section{Cervical Cancer Cells}

As shown in Figure 2, the percentages of apoptosis of Siha and Hela cells were decreased significantly by sevoflurane treatment and increased obviously after DDP treatment when compared with control group. However, statistical analysis showed that there was no significant difference between the DDP group and sevoflurane combined with DDP group. These results suggested that sevofluraneinhibited cell apoptosis but did not influence the promotion of DDP on the apoptosis of cervical cancer cells.

The result of proteins revealed that the expression of BCL-2 proteins was upregulated in Siha and Hela cells after sevoflurane treatment and downregulated significantly after DDP treatment. In addition, the expression of BAX was significantly reduced after sevoflurane treatment, while
DDP treatment significantly increased its expression. But no significant difference was indicated in the expressions of both BCL-2 and BAX proteins between the DDP and sevoflurane combined DDP group, which further demonstrated sevoflurane-inhibited cell apoptosis but had no influence on the promotional effect of DDP treatment in the process of apoptosis on cervical cancer cells.

\section{The Effect Of Sevoflurane And Sevoflurane Combined With DDP On The Cell Migration And Migration- Related Protein Ezrin And MMP2 In Cervical Cancer Cells}

The effect of sevoflurane or/and DDP on cervical cancer cells migration was assessed by determining the migration area by using the wound healing assay. As shown in Figure 3, compared with the control group, treatment of sevoflurane was significantly increased the migration area of cells at 24 hrs post-treatment, while the treatments of DDP were obviously decreased the migration area. Nevertheless, there was no statistical difference between the DDP group and sevoflurane combined with DDP group, which indicates that the inhibitory effect of DDP was not affected by the promotion of sevoflurane on the migration of cervical cancer cells. 
Siha NC

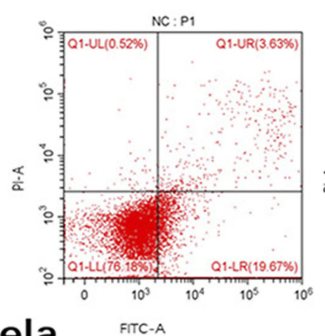

Hela firc-A

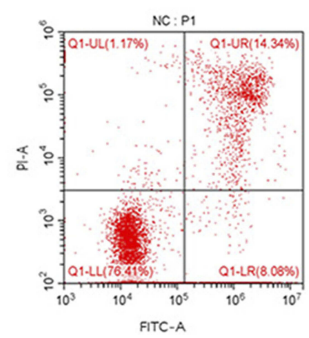

SEV

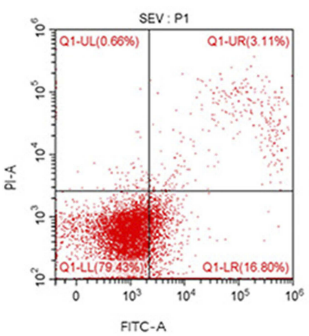

FITC-A

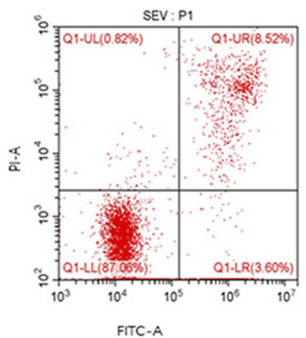

DDP

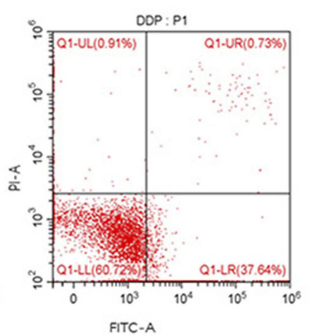

FITC-A

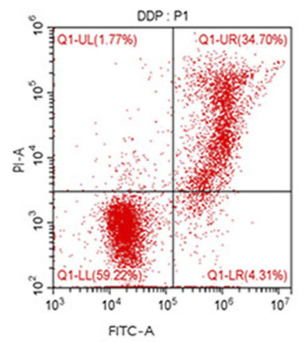

SEV+DDP
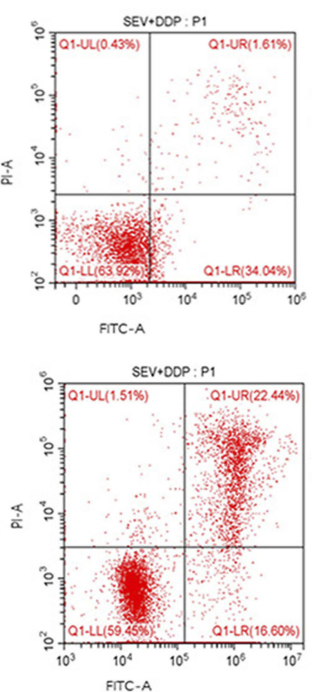

A

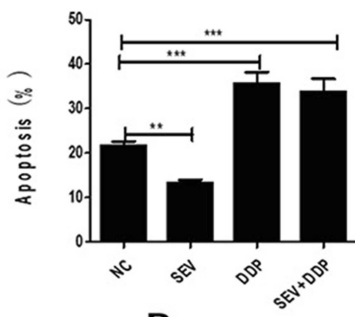

B

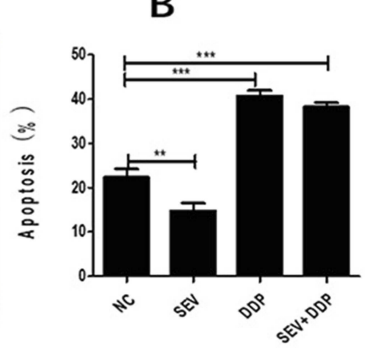

Siha

\section{BCL2}

BAX

\section{$\beta$-actin}

DDP

Sev

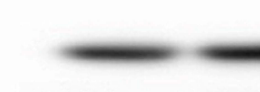

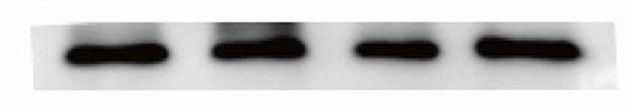

$+\quad+$

$+$

C

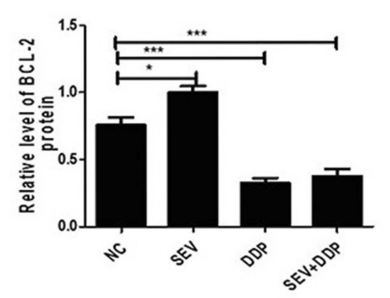

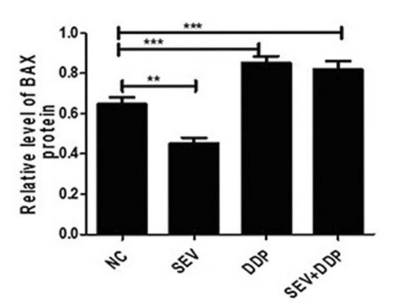

BCL2

BAX

$\beta$-actin

DDP

Sev

Hela
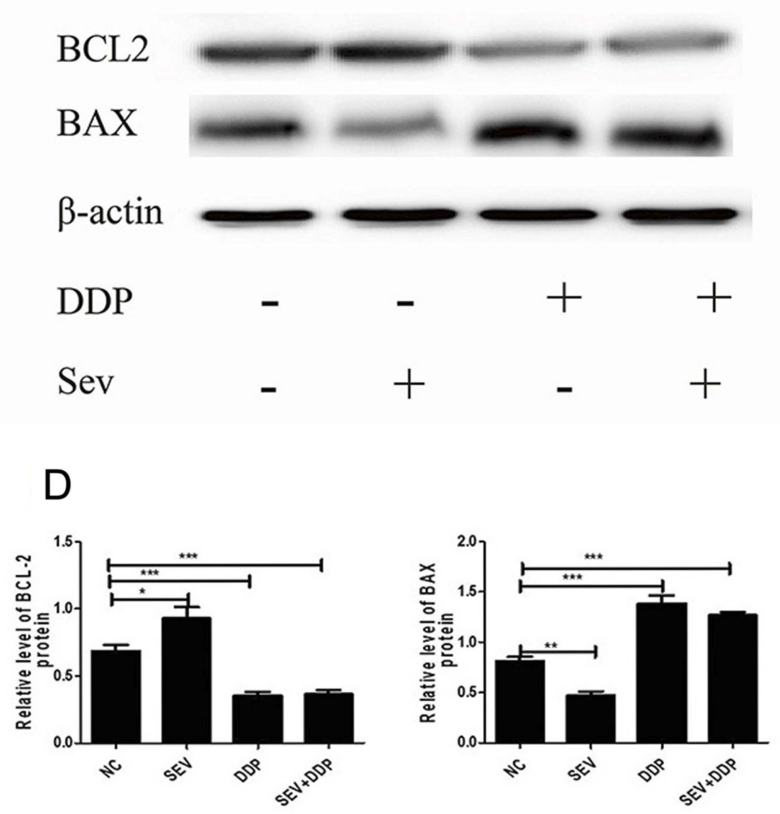

Figure 2 The effect of sevoflurane and sevoflurane combined with DDP on the cell apoptosis and apoptotic-related protein BCL-2 and BAX in cervical cancer cells. Siha and Hela cells were treated with sevoflurane, DDP, or the co-treatment of sevoflurane and DDP. The apoptosis percentages were evaluated by flow cytometry. The bars represent apoptosis percentages of each group. Data of Siha (A) and Hela (B) are shown as means and standard deviation of three independent experiments. The expressions of BCL-2 and BAX were determined by Western blot. The data of Siha (C) and Hela (D) are presented as relative expression level of $B C L-2$ and $B A X$ proteins normalized to $\beta$-actin. Data are shown as means and standard deviation of three independent experiments. $* P<0.05$, $* * P<0.01 * * * P<0.001$.

The result of proteins showed that sevoflurane treatment upregulated both of the Ezrin and MMP2 proteins expression, while DDP treatment suppressed their expressions significantly in Siha and Hela cells. In view of the levels of Ezrin and MMP2 protein, there was no statistical difference between DDP group and sevoflurane combined with DDP group. These results further demonstrated that sevoflurane promoted cell migration while had no effect on the inhibition of DDP treatment in the process of migration on cervical cancer cells. 

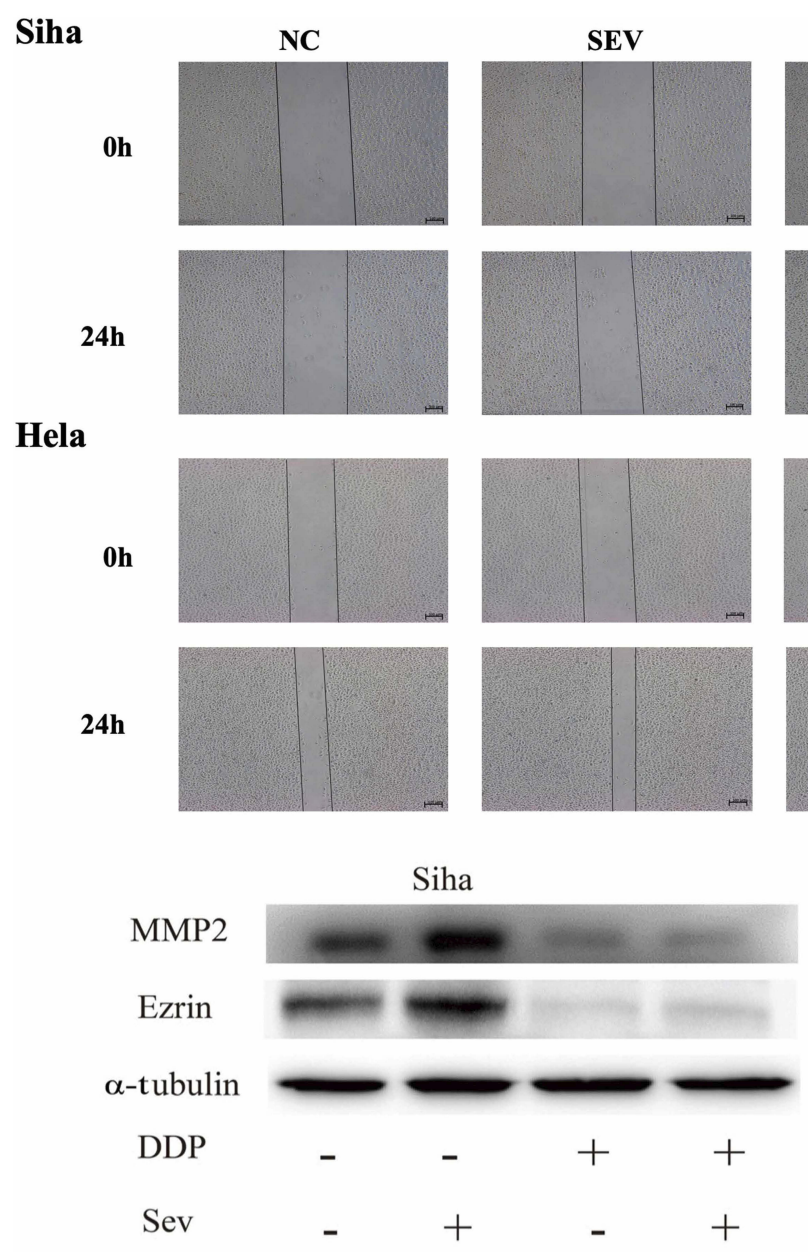

$\mathrm{C}$
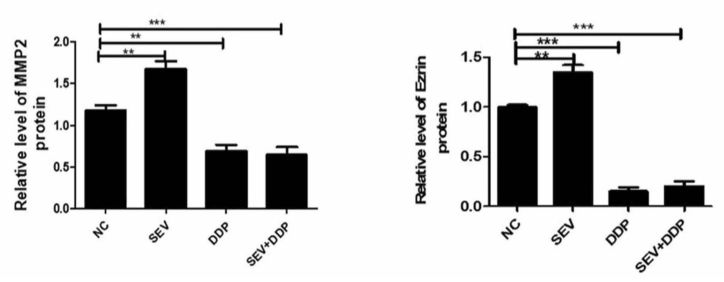
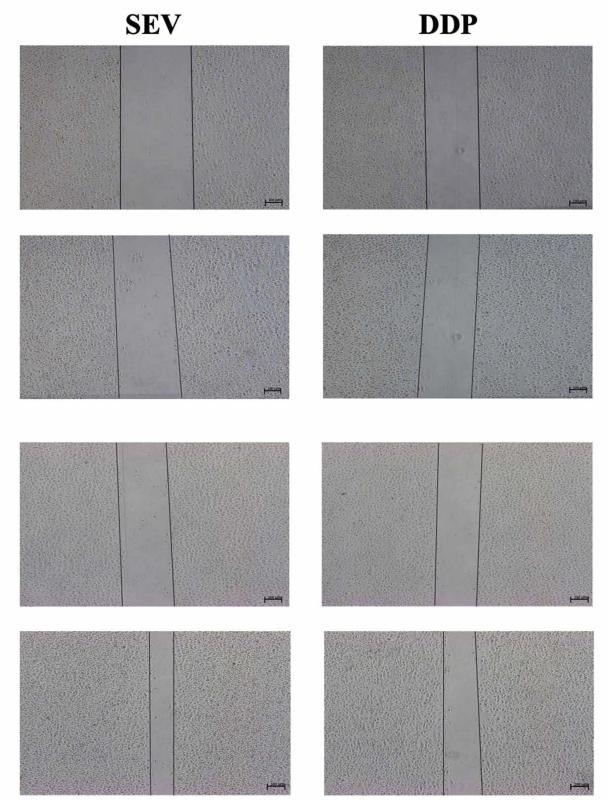
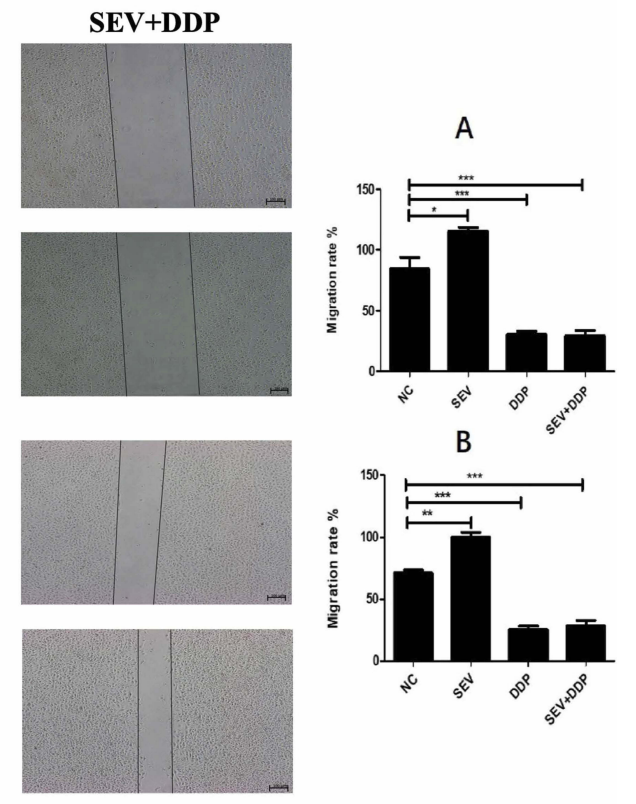

Hela

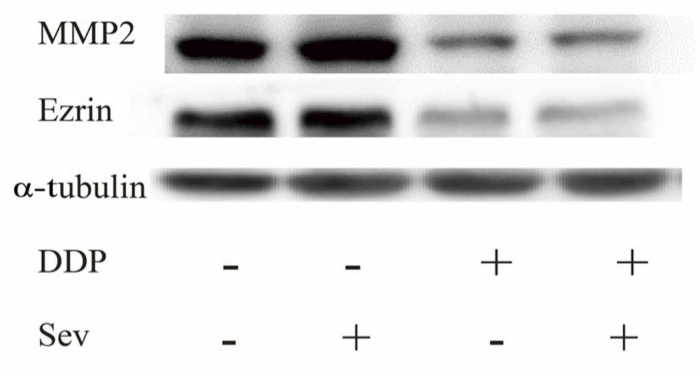

$\mathrm{D}$
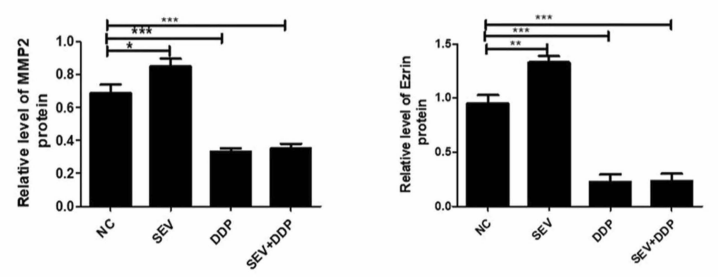

Figure 3 The effect of sevoflurane and sevoflurane combined with DDP on the cell migration and migration-related protein Ezrin and MMP2 in cervical cancer cells. Siha and Hela cells were treated with sevoflurane, DDP, or the co-treatment of sevoflurane and DDP. Migrating area from the initial wound was measured from images taken. Scratch areas of each group were analyzed in $0 \mathrm{~h}$ and $24 \mathrm{~h}$ treatment using Image). The bars represent migrating area of each group. Data of Siha (A) and Hela (B) are shown as means and standard deviation of three independent experiments. The expressions of Ezrin and MMP2 protein were determined by Western blot. The data of Siha (C) and Hela (D) are presented as relative expression level of Ezrin and MMP2 proteins normalized to $\boldsymbol{\alpha}$-tubulin. Data are shown as means and standard deviation of three independent experiments. $* P<0.05$, $* * P<0.01$, $* * * P<0.001$.

\section{The Effect Of Sevoflurane And}

\section{Sevoflurane Combined With DDP On} The Expression Of Drug ResistanceAssociated Proteins MRPI, LRP, GST-ח And P-gp In Cervical Cancer Cells

Overexpression of LRP, MRP1, GST- $\pi$ and P-gp played important roles in chemoresistance. To explore the effects of sevoflurane on cisplatinum resistance in cervical cancer cells, LRP, MRP1, GST- $\pi$ and P-gp proteins were investigated in the cells after sevoflurane or/and DDP treatment. As Figure 4 showed, the expressions of LRP and MRP1 were almost equal in the control group and sevoflurane group, and these two proteins were found to be almost equivalently down-regulated in the DDP group and DDP combined sevoflurane group. Moreover, the expression of GST- $\pi$ and P-gp proteins was not affected by sevoflurane or/and DDP treatment. In view of the LRP, MRP1, GST- $\pi$ 


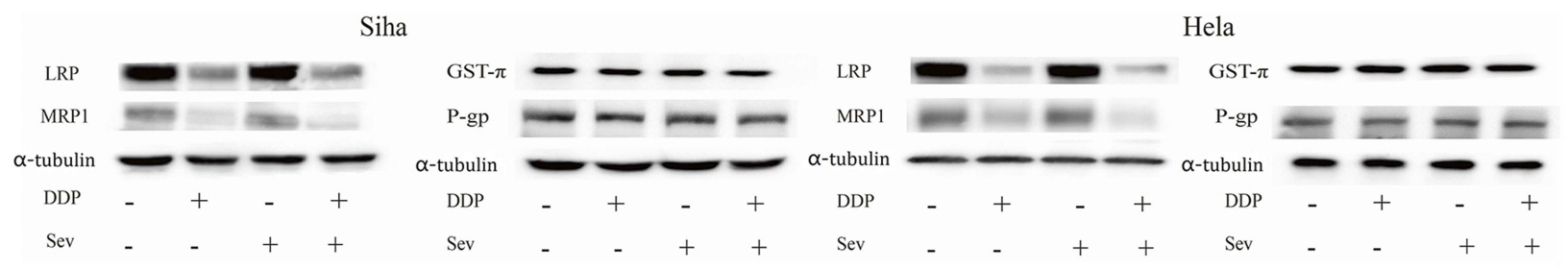

A
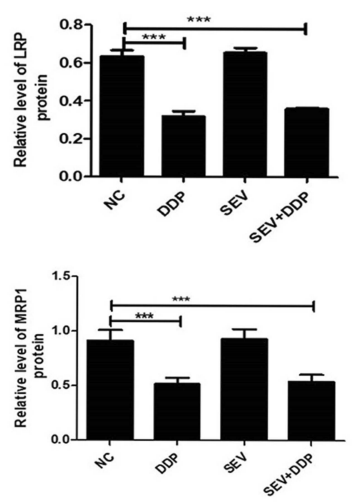

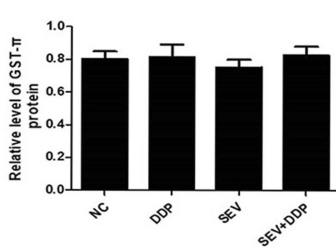

B

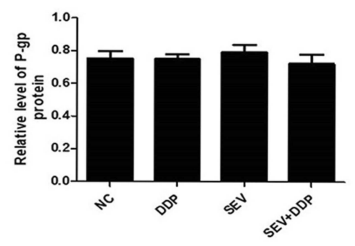

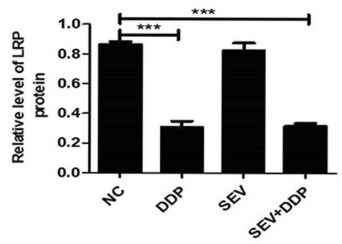
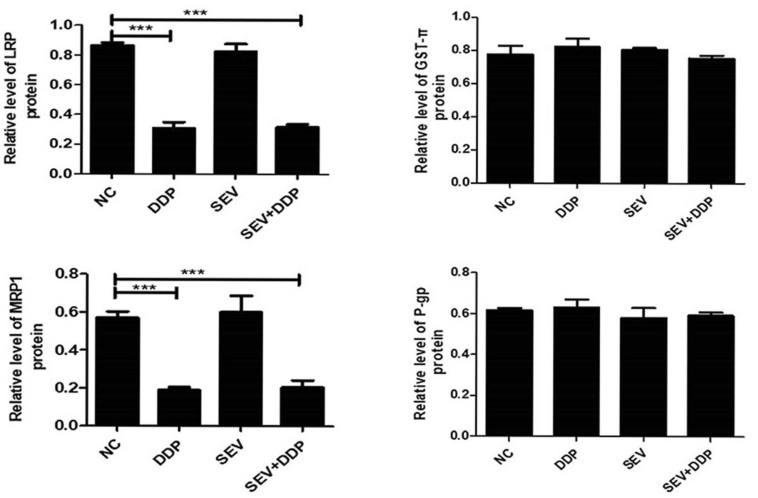

Figure 4 The effect of sevoflurane and sevoflurane combined with cisplatin (DDP) on the expression of drug resistance-associated proteins MRPI, LRP, GST- $\Pi$ and P-gP in cervical cancer cells. Siha and Hela cells were treated with sevoflurane, DDP, or the co-treatment of sevoflurane and DDP. The expressions of MRPI, LRP, GST- $\Pi$ and P-gP were determined by Western blot. The data of Siha $(\mathbf{A})$ and Hela (B) are presented as relative expression level of MRPI, LRP, GST-ח and P-gP proteins normalized to Tubulin. Data are shown as means and standard deviation of three independent experiments. $* * * P<0.001$.

and P-gp protein expression in these four treatment groups, it suggests that sevoflurane had no impact on the resistance of cervical cancer cells to DDP.

\section{Discussion}

Hysterectomy remains the standard treatment modality for patients with early-stage cervical cancer. ${ }^{12}$ Sevoflurane, a common volatile anesthetic, is extensively used in surgical oncology. Despite the primary surgical resection is often curative for these patients, the recurrence after surgery is inevitable among women with several high-risk factors including anesthetics administration. ${ }^{13-15}$ Hence, it is essential for these patients to receive an adjuvant DDPbased chemotherapy with or without radiotherapy postoperatively. ${ }^{16,17}$ The resistance of cancer cells to cisplatin significantly reduces the efficacy of DDP in chemotherapy. ERCC1-XPF, as a heterodimer, plays an important role in removing DNA damage caused by DDP. ${ }^{18,19}$ In addition, there are evidences that the use of narcotic drugs may also contribute to cisplatin resistance. Of note, though differential effects of sevoflurane on the chemosensitivity have been reported in lung ${ }^{11}$ and renal cell carcinomas, ${ }^{9}$ such effect of sevoflurane in cervical cancer remains to be elucidated. In this study, we first observed that sevoflurane promoted the proliferation and migration and inhibited the apoptosis, while had no effects on the sensitivity to DDP in cervical cancer cells.

Cell proliferation is considered as a vital indicator for cell survival or death when exposing to various conditions. ${ }^{20}$ In our study, we first demonstrated that sevoflurane alone exerted a positive effect on the proliferation of SiHa and HeLa cells, but had no influence on the inhibitory effect of DDP on the proliferation of cervical cancer cells.

BCL-2 protein-mediated apoptosis blockade contributes to malignant transformation in multiple human cancers, whereas Bax protein enhances the permeability of the mitochondrial outer membrane and induces release of cytochrome c and consequently promotes apoptosis, which can be counteracted by BCL- $2 .^{21}$ In the present study, we first observed that sevoflurane treatment apparently suppressed the apoptosis of $\mathrm{SiHa}$ and HeLa cells, as confirmed by Western blot analyses with a remarkable elevation of BCL-2 and sharp decline of Bax expression in both cell lines. Notably, accumulated evidences have revealed that the sensitivity to DDP is conferred by suppression of BCL-2 expression in various types of human cancers. $^{22,23}$ Thus, the cytotoxic effect caused by DDP in cervical cancer cells might be weakened by sevoflurane via increasing the expression of BCL-2. However, neither 
the apoptotic rate nor the level of related proteins (BCL-2 and BAX) was significantly changed in DDP-treated $\mathrm{SiHa}$ and Hela cells after sevoflurane contact, demonstrating that pro-apoptotic function induced by DDP was not altered by sevoflurane.

The metastasis of cancer is a complex process involving the dissemination of tumor from the primary site to distant organs. ${ }^{24}$ Numerous scientific and clinical evidences have shown that Ezrin and Matrix metalloproteinase 2 (MMP2) proteins are important structural molecule controlling cell adhesion and invasive properties of cancer cells, ${ }^{25,26}$ including cervical cancer. ${ }^{25}$ In line with this notion, our study first detected that sevoflurane promoted the migration of both $\mathrm{SiHa}$ and HeLa cells, which was mediated by upregulating the expression of Ezrin and MMP2 proteins. Nevertheless, it is worth noting that cancer metastasis plays a crucial role in generating drug resistance, in which Ezrin and MMP2 were involved. ${ }^{25-27}$ But fortunately, there was no significant difference found between DDP and DDP combined with sevoflurane treatment groups, supporting that DDP-induced suppression on cervical cancer cells migration was not affected by sevoflurane.

Multidrug resistance-related protein 1 (MRP1) and P-glycoprotein (P-gp) are the best-characterized transport proteins and commonly protect cells by effluxing chemotherapeutic agents, whose high expression in cancer cells can lead to a decrease in intracellular platinum concentration. ${ }^{28-30}$ In this study, we found that the level of MRP1 and P-gp was not changed in sevoflurane group, suggesting that sevoflurane administration alone had no effect on chemosensitivity in cervical cancer cells. Additionally, the expression of MRP1 was almost equally downregulated by DDP and DDP combined with sevoflurane treatment, demonstrating that the DDP resistance of $\mathrm{SiHa}$ and HeLa cells was not influenced by the action of sevoflurane. Interestingly, the expression of P-gp did not change in the application of DDP and DDP combined with sevoflurane treatment. Hence, we suggested although there are several resemblances in the biochemical and pharmacological properties of MRP1 and P-gp, the major protein involved in cisplatinum resistance in Siha and Hela cells is MRP1 rather than P-gp. Similar results were reported in one study from Cole et $\mathrm{al},{ }^{31}$ they identified an overexpression of MRP1 rather than P-gp in doxorubicin-resistant HeLa cell lines.

Lung-resistance-related protein (LRP) and glutathioneS-transferase- $\pi$ (GST- $\pi$ ) are two kinds of non-transport proteins which are generally considered to be associated with multidrug resistance (MDR) of various tumors. ${ }^{32-34}$ Our results showed that the level of LRP and GST- $\pi$ were not altered by sevoflurane, indicating that application of sevoflurane alone had no influence on chemosensitivity in both SiHa and HeLa cells. However, the expression of GST$\pi$ was not changed after the action of DDP or DDP combined with sevoflurane treatment. This result is similar to the findings of Li et al, ${ }^{35}$ with a low expression of LRP only in DDP-resistant ovarian cancer cells rather than GST- $\pi$.

Little effort has been made to explore the effects of sevoflurane on the biological behavior and chemosensitivity in various types of cancer cells. For example, Ciechanowicz et $\mathrm{al}^{9}$ found that sevoflurane promoted the proliferation, migration and reduced the DDP sensitivity in kidney cancer cells, which were reversed by $T G F-\beta$ and $O P N$ knockdown. Similar results were also observed in human breast cancer, ${ }^{7}$ colon cancer $^{36}$ and hepatocellular carcinoma cells. ${ }^{37}$ Conversely, two groups revealed that, in human lung adenocarcinoma cells, the metastatic potential was significantly inhibited while the anticancer effects of DDP were enhanced after sevoflurane exposure, respectively, which was associated with upregulation of nuclear Smad3 and downregulation of XIAP, Survivin and MMPs. ${ }^{5,9}$ In fact, the cytotoxic effect of the combined application of DDP and sevoflurane on a variety of cells has also been investigated in vivo. ${ }^{8}$ It showed that sevoflurane administration in mice induced obvious genotoxic effect in peripheral blood leucocytes, liver, kidney, brain and Ehrlich ascites tumour cells, while a synergistic effect of combined treatments was not expressed in brain and Ehrlich ascites tumor cells. Thereafter, another in vivo research ${ }^{10}$ reported that apoptosis in Ehrlich ascites tumor cells was not affected under application of sevoflurane alone but was significantly attenuated after the co-treatment of sevoflurane and DDP. From the above, we suggested that sevoflurane may exhibit dual effects on behavior functions and chemosensitivity in different human cancers in a tissue and cellular context-dependent manner.

There are still some limitations that need to be interpreted cautiously in this study. First, diverse volatile and intravenous (such as propofol) anesthetics are usually used in combination in clinical practice. Thus, it is necessary to investigate the influence of sevoflurane and other types of anesthetics on cervical cancer cell lines especially with cisplatinum-resistance in the future. Second, experiments in our study were all designed in vitro, which should be conducted in vivo in the future. Third, further studies need 
to be carried out to explore the potential molecular mechanisms or signaling pathways regulating the effect of sevoflurane on cervical cancer cell lines which are obtained from cisplatin-resistant patients.

\section{Conclusion}

In conclusion, despite the limitations, results of our study favor that the use of sevoflurane can affect the proliferation, migration and apoptosis of cervical cancer cells. Sevoflurane promotes the proliferation and migration of Siha and Hela cell lines, while it exerts an inhibitory function on the apoptosis of cells. But sevoflurane did not attenuate the cisplatinum sensitivity in cervical cancer cells.

\section{Acknowledgements}

This work was supported by a grant from the National Research Foundation of Health and Family Planning Commission - the Medical Science and Technology Project of Zhejiang Province (WKJ-ZJ-1528) and Project of Wenzhou science and Technology Bureau (Y20150197).

\section{Author contributions}

X.Q. Zhu and R.Y. Li conceived and designed the study. F. Xue, Y.C. Xu and W.W. Zhang acquired the data by did the experiments. Y. Z. Song and Y.C. Xu analyzed and interpreted the data. F. Xue, Y.C. Xu and W.W. Zhang drafted the article. X.Q. Zhu, R.Y. Li and Y.Z. Song revised the important intellectual content of manuscript. All authors contribute to data analysis, drafting and revising the paper and agree to be accountable for all aspects of the work in ensuring that questions related to the accuracy or integrity of any part of the work are appropriately investigated and resolved. All authors have read and approved the final version to be published.

\section{Disclosure}

The authors report no conflicts of interest in this work.

\section{References}

1. Siegel RL, Miller KD, Jemal A. Cancer statistics, 2019. CA Cancer J Clin. 2019;69(1):7-34. doi:10.3322/caac.21551

2. Pfaendler KS, Tewari KS. Changing paradigms in the systemic treatment of advanced cervical cancer. Am J Obstet Gynecol. 2016;214 (1):22-30. doi:10.1016/j.ajog.2015.07.022

3. Tangjitgamol S, Katanyoo K, Laopaiboon M, Lumbiganon $P$, Manusirivithaya S, Supawattanabodee B. Adjuvant chemotherapy after concurrent chemoradiation for locally advanced cervical cancer. Cochrane Database Syst Rev. 2014;12:CD010401.

4. Xiang M, Kidd EA. Benefit of cisplatin with definitive radiotherapy in older women with cervical cancer. J Natl Compr Canc Netw. 2019;17 (8):969-975. doi:10.6004/jncen.2019.7289
5. Liang H, Gu M, Yang C, Wang H, Wen X, Zhou Q. Sevoflurane inhibits invasion and migration of lung cancer cells by inactivating the p38 MAPK signaling pathway. J Anesth. 2012;26(3):381-392. doi:10.1007/s00540-011-1317-y

6. Muller-Edenborn B, Roth-Z'graggen B, Bartnicka K, et al. Volatile anesthetics reduce invasion of colorectal cancer cells through downregulation of matrix metalloproteinase-9. Anesthesiology. 2012;117 (2):293-301. doi:10.1097/ALN.0b013e3182605df1

7. Ecimovic P, McHugh B, Murray D, Doran P, Buggy DJ. Effects of sevoflurane on breast cancer cell function in vitro. Anticancer Res. 2013;33(10):4255-4260.

8. Brozovic G, Orsolic N, Knezevic F, et al. Evaluation of DNA damage in vivo induced by combined application of cisplatin and sevoflurane. Eur J Anaesthesiol. 2008;25(8):642-647. doi:10.1017/S0265021508004171

9. Ciechanowicz S, Zhao H, Chen Q, et al. Differential effects of sevoflurane on the metastatic potential and chemosensitivity of nonsmall-cell lung adenocarcinoma and renal cell carcinoma in vitro. $\mathrm{Br}$ $J$ Anaesth. 2018;120(2):368-375. doi:10.1016/j.bja.2017.11.066

10. Brozovic G, Orsolic N, Knezevic F, et al. Genotoxicity and cytotoxicity of cisplatin treatment combined with anaesthetics on EAT cells in vivo. Onkologie. 2009;32(6):337-343. doi:10.1159/000218066

11. Liang H, Wang H, Liu H, Wen X, Zhou Q, Yang C. The effects of combined treatment with sevoflurane and cisplatin on growth and invasion of human adenocarcinoma cell line A549. Biomed Pharmacother. 2013;67(6):503-509. doi:10.1016/j.biopha.2013.03.005

12. Cibula D, Potter R, Planchamp F, et al. The European Society of Gynaecological Oncology/European Society for Radiotherapy and Oncology/European Society of Pathology guidelines for the management of patients with cervical cancer. Int J Gynecol Cancer. 2018;28 (4):641-655. doi:10.1097/IGC.0000000000001216

13. Chemoradiotherapy for Cervical Cancer Meta-Analysis Collaboration. Reducing uncertainties about the effects of chemoradiotherapy for cervical cancer: a systematic review and meta-analysis of individual patient data from 18 randomized trials. J Clin Oncol. 2008;26 (35):5802-5812. doi:10.1200/JCO.2008.16.4368

14. Tohme S, Simmons RL, Tsung A. Surgery for cancer: a trigger for metastases. Cancer Res. 2017;77(7):1548-1552. doi:10.1158/00085472.CAN-16-1536

15. Buggy DJ, Hemmings HC. Special issue on anaesthesia and cancer. Br J Anaesth. 2014;113(Suppl 1):i1-i3. doi:10.1093/bja/aeu261

16. Takekuma M, Kasamatsu Y, Kado N, et al. The issues regarding postoperative adjuvant therapy and prognostic risk factors for patients with stage I-II cervical cancer: a review. J Obstet Gynaecol Res. 2017;43(4):617-626. doi:10.1111/jog.13282

17. Li L, Song X, Liu R, et al. Chemotherapy versus radiotherapy for FIGO stages IB1 and IIA1 cervical carcinoma patients with postoperative isolated deep stromal invasion: a retrospective study. $B M C$ Cancer. 2016;16:403. doi:10.1186/s12885-016-2447-2

18. Jokic M, Vlasic I, Rinneburger M, et al. Ercc1 deficiency promotes tumorigenesis and increases cisplatin sensitivity in a Tp53 context-specific manner. Mol Cancer Res. 2016;14(11):1110-1123. doi:10.1158/15417786.MCR-16-0094

19. Hamilton G, Rath B. Pharmacogenetics of platinum-based chemotherapy in non-small cell lung cancer: predictive validity of polymorphisms of ERCC1. Expert Opin Drug Metab Toxicol. 2018;14 (1):17-24. doi:10.1080/17425255.2018.1416095

20. Adan A, Kiraz Y, Baran Y. Cell proliferation and cytotoxicity assays. Curr Pharm Biotechnol. 2016;17(14):1213-1221. doi:10.2174/ 1389201017666160808160513

21. Beroukhim R, Mermel CH, Porter D, et al. The landscape of somatic copy-number alteration across human cancers. Nature. 2010;463 (7283):899-905. doi:10.1038/nature08822

22. Liu J, Yang L, Zhang J, et al. Knock-down of NDRG2 sensitizes cervical cancer Hela cells to cisplatin through suppressing Bcl-2 expression. BMC Cancer. 2012;12:370. doi:10.1186/1471-2407-12370 
23. Chen X, Zhang Y, Shi Y, et al. MiR-873 acts as a novel sensitizer of glioma cells to cisplatin by targeting Bcl-2. Int J Oncol. 2015;47 (4):1603-1611. doi:10.3892/ijo.2015.3143

24. Clucas J, Valderrama F. ERM proteins in cancer progression. J Cell Sci. 2014;127(Pt 2):267-275. doi:10.1242/jcs.133108

25. Kessenbrock K, Plaks V, Werb Z. Matrix metalloproteinases: regulators of the tumor microenvironment. Cell. 2010;141(1):52-67. doi:10.1016/j.cell.2010.03.015

26. Ou-Yang M, Liu HR, Zhang Y, Zhu X, Yang Q. ERM stable knockdown by siRNA reduced in vitro migration and invasion of human SGC-7901 cells. Biochimie. 2011;93(5):954-961. doi:10.1016/j. biochi.2011.01.017

27. Qin R, Cao L, Wang J, Liu J. Promoter methylation of ezrin and its impact on the incidence and prognosis of cervical cancer. Cell Physiol Biochem. 2018;50(1):277-287. doi:10.1159/000494005

28. Conseil G, Arama-Chayoth M, Tsfadia Y, Cole SPC. Structureguided probing of the leukotriene $\mathrm{C}$ binding site in human multidrug resistance protein 1 (MRP1; ABCC1). FASEB J. 2019;16.

29. Abdallah HM, Al-Abd AM, El-Dine RS, El-Halawany AM. P-glycoprotein inhibitors of natural origin as potential tumor chemo-sensitizers: a review. $J$ Adv Res. 2015;6(1):45-62. doi:10.1016/j.jare.2014.11.008

30. Krishnakumar S, Mallikarjuna K, Desai N, et al. Multidrug resistant proteins: P-glycoprotein and lung resistance protein expression in retinoblastoma. Br J Ophthalmol. 2004;88(12):1521-1526. doi:10.1136/ bjo.2004.047928
31. Cole SP, Bhardwaj G, Gerlach JH, et al. Overexpression of a transporter gene in a multidrug-resistant human lung cancer cell line. Science. 1992;258(5088):1650-1654. doi:10.1126/science.1360704

32. Dalton WS, Scheper RJ. Lung resistance-related protein: determining its role in multidrug resistance. J Natl Cancer Inst. 1999;91 (19):1604-1605. doi:10.1093/jnci/91.19.1604

33. Bouhamyia L, Chantot-Bastaraud S, Zaidi S, et al. Immunolocalization and cell expression of lung resistance-related protein (LRP) in normal and tumoral human respiratory cells. J Histochem Cytochem. 2007;55 (8):773-782. doi:10.1369/jhc.7A7176.2007

34. Mao ZP, Zhao LJ, Zhou SH, Liu MQ, Tan WF, Yao HT. Expression and significance of glucose transporter-1, P-glycoprotein, multidrug resistance-associated protein and glutathione S-transferase-pi in laryngeal carcinoma. Oncol Lett. 2015;9(2):806-810. doi:10.3892/ ol.2014.2752

35. Li L, Luan Y, Wang G, et al. Development and characterization of five cell models for chemoresistance studies of human ovarian carcinoma. Int J Mol Med. 2004;14(2):257-264.

36. Sugimoto H, Kawaraguchi Y, Nomura Y, et al. Exposure to $1 \%$ sevoflurane for $6 \mathrm{hrs}$ enhances proliferation of human colon cancer cells. Masui. 2015;64(4):357-361.

37. Nishiwada T, Kawaraguchi Y, Uemura K, Sugimoto H, Kawaguchi M. Effect of sevoflurane on human hepatocellular carcinoma HepG2 cells under conditions of high glucose and insulin. J Anesth. 2015;29 (5):805-808. doi:10.1007/s00540-015-2025-9

\section{Publish your work in this journal}

Drug Design, Development and Therapy is an international, peerreviewed open-access journal that spans the spectrum of drug design and development through to clinical applications. Clinical outcomes, patient safety, and programs for the development and effective, safe, and sustained use of medicines are a feature of the journal, which has also been accepted for indexing on PubMed Central. The manuscript management system is completely online and includes a very quick and fair peer-review system, which is all easy to use. Visit http://www. dovepress.com/testimonials.php to read real quotes from published authors. 